\title{
MODELOS MATEMÁTICOS: UM MECANISMO DE GESTÃO AMBIENTAL.
}

Anderson da Silva Palmeira1; Eder Johnson de Área Leão Pereira2; Carlos César Ribeiro Santos2; Paulo Henrique Farias Xavier2; André Luiz Santos da Soledade2; Davidson Martins Moreira2.

1,2Centro Universitário SENAI CIMATEC, Av. Orlando Gomes 1845 - Piatã; Salvador/Bahia; andersonpalmeira@icloud.com

Resumo: O presente artigo apresenta a importância dos modelos matemáticos de gestão ambiental como mecanismo de mitigação de emissões antrópicas. O trabalho justifica-se pela importância em apresentar os modelos matemáticos de gestão ambiental como mecanismo de mitigação de emissões antrópicas. Os estudos foram fundamentados em ideias e pressupostos de teóricos que apresentam significativa importância na definição e construção dos conceitos discutidos nesta análise: convenções mundiais que abordam o tema de gestão ambiental. Para tal, realizou-se uma revisão na literatura. Por fim, o problema apresentado nessa pesquisa consistiu em vincular os pactos de gestão ambiental aos modelos matemáticos, pois na literatura os assuntos são abordados de forma separada.

Palavras-Chave: Gestão Ambiental; Porto de Aratu; Modelos Matemáticos; Acordo de Paris.

\section{MATHEMATICAL MODELS: A MECHANISM FOR ENVIRONMENTAL MANAGEMENT.}

Abstract: This paper presents the importance of mathematical models of environmental management as an anthropogenic emission mitigation mechanism. The paper is justified by the importance of presenting the mathematical models of environmental management as an anthropogenic emissions mitigation mechanism. The studies were based on ideas and assumptions of theorists that have significant importance in the definition and construction of the concepts discussed in this analysis: world conventions that address the issue of environmental management. To this end, a literature review was performed. Finally, the problem presented in this research consisted in linking environmental management pacts to mathematical models, because in the literature the subjects are approached separately.

Keywords: Environmental management; Aratu Port; Mathematical models; Paris agreement.

\section{INTRODUÇÃO}

A aplicabilidade da Modelagem Matemática, para os autores [27], na descrição e interpretação de dados específicos, constitui uma ferramenta valiosa para a solução de problemas reais, bem como, para a fundamentação de decisões. Por isso, torna-se interessante envolver a matemática em questões relacionadas com o Meio Ambiente.

Os modelos matemáticos, para [31], permitem de certa forma avaliar a contribuição de uma única fonte à carga poluidora, ou seja, administrar liberações emergenciais e analisar áreas de riscos. Assim, na gestão ambiental, os modelos são usados para: indicar áreas com maiores concentrações de poluentes que possam acelerar a degradação de materiais, indicar locais para instalação de estações de monitoramento; dar suporte a planos de ação para redução de emissões de poluentes; prever impactos de novos empreendimentos; auxiliar na verificação das tecnologias industriais ou propostas de 
adequação quanto ao atendimento à legislação vigente, entre outras finalidades. Já [2], afirmam que a valoração econômica dos recursos ambientais é fundamental para fixar a compensação financeira, correspondente aos impactos ambientais causados por empreendimentos ocorridos na fase de licenciamento ou já instalados.

Diante o exposto, o presente artigo intitulado 'Modelos Matemáticos: um mecanismo para gestão ambiental' visa apresentar a importância dos modelos matemáticos de gestão ambiental como mecanismo de mitigação de emissões antrópicas, assim como fundamentar a evolução dos pactos na área de gestão ambiental; apresentar fundamentos da importância dos modelos matemáticos para gestão ambiental e por fim, apresentar a aplicação da gestão ambiental no Porto de Aratu no município de Candeias - Bahia. No âmbito desse estudo será abordado modelos matemáticos voltados para gestão ambiental, visto que é um tema atual e importante para a humanidade. Salientase que o meio ambiente é vital para o ser humano e requer um processo de gestão para que possamos ter condições ambientais favoráveis e fatores naturais disponíveis para as gerações futuras se desenvolverem e darem continuidade a vida humana no planeta Terra.

Este trabalho será fundamentado em ideias e pressupostos de teóricos que apresentam significativa importância na definição e construção dos conceitos discutidos nesta análise: convenções mundiais que abordam o tema de gestão ambiental. Para tal, realizou-se uma revisão na literatura.

Assim sendo, o trabalho transcorrerá a partir do método conceitual-analítico, visto que se utilizam conceitos e ideias de outros autores, semelhantes com os nossos objetivos, para a construção de uma análise científica sobre o nosso objeto de estudo. $O$ presente estudo justifica-se pela importância em apresentar os modelos matemáticos de gestão ambiental como mecanismo de mitigação de emissões antrópicas. Este artigo é dividido em três tópicos: o primeiro tópico aborda um breve histórico dos momentos importantes para a formulação da política ambiental internacional, em seguida o segundo tópico apresenta a importância dos modelos como mecanismo de gestão ambiental, por fim o terceiro tópico apresenta uma aplicação da gestão da emissões no Porto de Aratu utilizando modelagem matemática através da Rede de Monitoramento da Qualidade do Ar e Meteorologia (RMAR).

\section{CONTEXTUALIZAÇÃO}

O Acordo de Paris é um compromisso internacional discutido entre 195 países com o objetivo de minimizar as consequências do aquecimento global. Ele foi adotado durante a Conferência das Partes - COP 21, em Paris, no ano de 2015. Seu principal objetivo pautou-se exclusivamente com o compromisso no sentido de manter o aumento da temperatura média global em bem menos de $2^{\circ} \mathrm{C}$ acima dos níveis pré-industriais e de envidar esforços para limitar o aumento da temperatura a $1,5^{\circ} \mathrm{C}$ acima dos níveis préindustriais. Em 1972 aconteceu na Suécia, a Conferência da ONU (Organização das Nações Unidas) sobre Meio Ambiente, considerada um momento histórico para política internacional do Meio Ambiente. De acordo com [8], foram quatro os principais fatores que motivaram, à época, a decisão de realizar uma conferência mundial sobre a proteção do meio ambiente.

No ano de 1972, realizou-se a Conferência da ONU (Organização das Nações Unidas) também conhecida como a Conferência de Estocolmo com a participação de 113 países, entre eles o Brasil, e de 400 organizações governamentais e não-governamentais cujo objetivo era de conscientizar a sociedade a melhorar a relação com o meio ambiente 
e assim atender as necessidades da população presente sem comprometer as gerações futuras. Na Conferência originou-se uma declaração que ficou conhecida como Declaração de Estocolmo que destaca em seu preâmbulo sete pontos principais e vinte e seis princípios que oferece aos povos do mundo inspiração e guia para preservar e melhorar o meio ambiente. No ponto dois do preâmbulo, a declaração ressalta a necessidade de um ambiente equilibrado para a qualidade de vida humana, ratifica a responsabilidade da sociedade e dos governos em buscar uma harmonia entre crescimento econômico e proteção ao meio ambiente, conforme [8].

No início da década de 1980, a ONU retomou o debate das questões ambientais. Com a organização da Comissão Mundial sobre o Meio Ambiente e Desenvolvimento chefiada pela primeira-ministra da Noruega, Gro Harlem Brundtland. O objetivo da Comissão sobre o Meio Ambiente foi analisar os acontecimentos após a Conferência de Estocolmo e propor discussões para firmar acordos ambientais internacionais. $O$ resultado da comissão foi a elaboração de um relatório intitulado 'Nosso Futuro Comum', publicado em 1987. De acordo com [3]: 12 anos depois, ou seja, em 1992, a ONU concede espaço e amplia as discussões em busca de acordos ambientais, desta vez a cidade escolhida foi o Rio de Janeiro, sede da Conferência da ONU sobre Meio Ambiente e Desenvolvimento (UNCED), o evento ficou internacionalmente conhecido como Rio-92. De acordo com [5]: "[...] reuniu mais de 35 mil pessoas, entre elas 106 chefes de governos". Para [26] esse evento resultou: "[...] em cinco documentos: a Agenda 21, a Convenção do Clima, a Convenção da Biodiversidade, a Declaração do Rio e os Princípios sobre Florestas". Estes documentos contêm ou delineiam acordos internacionais que têm como objetivo modificar os sistemas antropogênicos em direção ao desenvolvimento sustentável. A Agenda 21 foi um documento de extrema importância, pois expõe acordos entre países e direciona a harmonia entre o meio ambiente $\mathrm{e}$ crescimento econômico.

Segundo [20]: "[...] Impulsionados pelas discussões e pelos resultados dessa Conferência, e seguindo a Agenda 21 Global, governo e sociedade deram início a um conjunto de ações de construção de Agendas 21, nos âmbitos nacional, regional e local". Outro documento da Rio-92 foi a Convenção do Clima, que tem o objetivo de acordo com [11]: Após a Rio-92, várias reuniões sobre o clima foram realizadas, uma em especial ocorrida no Japão em 1997, na ocasião foi ratificado o Protocolo de Quioto, que segundo [30]: "[...] com esse protocolo, foram estabelecidas metas de redução das emissões das fases do efeito estufa, que devem ser cumpridas até 2012 tendo como referências as emissões de 199 ". Os Estados Unidos, um país importantíssimo no cenário econômico mundial se negou a contribuir com a redução dos gases do efeito estufa.

Em 2002 foi realizada pela ONU, a Cúpula Mundial sobre Desenvolvimento Sustentável em Johanesburgo, na África do Sul, também conhecida como Rio+10 ou Cúpula da Terra II, o objetivo foi analisar os avanços da Agenda 21 e da Cúpula de 1992. Desta Cúpula de 2002, surgiram então, dois documentos, a Declaração de Johanesburgo e o Plano de Implementação. Dez anos depois da Rio+10 aconteceu a Conferência das Nações Unidas sobre Desenvolvimento Sustentável - Rio+20, realizada de 13 a 22 de junho de 2012, na cidade do Rio de Janeiro. Participaram da Rio+20, chefes de Estado e de Governo e a sociedade civil. Os dois temas principais que orientaram os debates na Rio+20 foram: a economia verde, no contexto do desenvolvimento sustentável e da erradicação da pobreza. Após vários debates, palestras e reuniões resultaram em um documento com o título: "O Futuro que Queremos", nele está reafirmado o compromisso com o desenvolvimento sustentável do nosso planeta, com a promoção de uma 
integração dos aspectos econômicos, social e ambientais, com meta de erradicação da pobreza utilizando a economia verde, mostrada no documento como uma ferramenta capaz de garantir o crescimento econômico e promover a sustentabilidade.

Entretanto alguns autores criticam as ideias sobre a relevância da Rio+20 para o desenvolvimento sustentável. Um dos pontos bastante criticado foi a ausência de discussões sobre os temas: o Clima e a Biodiversidade, considerados importantíssimos no cenário ambiental e a falta de clareza nas metas e acordos, como reforça [14].

De acordo com explicitado acima se percebe que as Convenções sobre o Meio Ambiente, ocorridas desde a década de 1960, têm se mostrado utópicas e cautelosas no que diz respeito a elaboração de metas e a exigência do cumprimento dessas metas para o desenvolvimento sustentável, fica claro que as mudanças climáticas e catástrofes naturais que acontecem com frequência em todo o mundo são vista como algo normal e os recursos naturais que são explorados ilegalmente não fosse chegar a um esgotamento futuro. A principal barreira para a efetivação de metas para o desenvolvimento sustentável é o fato desse tema está associado ao setor econômico e os países desenvolvidos resistem em assinar os acordos ambientais propostos para não comprometer sua economia, visto que o consumismo é cada vez maior e exploração de recursos naturais gera muito lucro.

Sobre o cenário ambiental brasileiro de acordo com [30]:[...] os movimentos ecológicos surgiram na década de 1970 e vêm se firmando aos poucos[...]. Foram criados organismos federais e estaduais voltados ao assunto, tais como o Ministério do Meio Ambiente (MMA), o Instituto de Meio Ambiente (Ibama) e a Secretária Especial do Meio Ambiente (Sema). Tornou-se obrigatório antes dos empreendimentos que utilizem recursos ambientais ou que tenha algum impacto ambiental relatório de licenciamento ambiental como o EIA - Estudos de Impactos Ambientais e RIMA - Relatório de Impacto ao Meio Ambiente. Como continuidade e objetivando tratar as lacunas sobre o tema, temos o acordo de Paris aceito por 195 países e seus termos.

O Acordo de Paris é uma aliança produzida ao longo do tempo, oficialmente vinculada a acordos ambientais que preexiste e define metas mundiais, como o de limite de mitigação de temperatura mundial e o de subsídio climático corroborando com os países em desenvolvimento. Também traz metas com limites de emissões de gases de efeito estufa para todos os países. Trata-se, de fato, de uma espécie de manual para a reorientação da economia mundial no rumo da descarbonização. O acordo foi bem recebido, mas sabe-se que o trabalho de fato começa agora. Atingir suas metas e evitar o caos climático depende de pactos muito mais robustos do que os anunciados pelos países antes da COP21.

O ano de 2015 foi histórico para a agenda de clima. Após duas décadas de negociações no âmbito da Convenção-Quadro das Nações Unidas sobre Mudança do Clima, a UNFCCC, e diante de intensa mobilização de cientistas, da sociedade civil, do setor privado, de governos nacionais e subnacionais e de lideranças religiosas, como o Papa Francisco, países de todo o mundo chegaram a um acordo efetivamente global para o enfrentamento das mudanças climáticas.

A Convenção finalmente entra em fase de plena implementação. A assinatura do acordo, em 2015, foi histórica por unir, pela primeira vez, quase todos os países do mundo em um pacto voltado às mudanças climáticas. Através do pacto celebrado pelo acordo de paris foram adotados mecanismos maleáveis com objetivo de cumprir o tratado. O acordo de Paris aumenta as obrigações de atenuação de emissões dos membros participantes, 
pois no protocolo de Quioto apenas os países desenvolvidos possuíam obrigações de redução na emissão de gases do efeito estufa, hoje todos os países membros do acordo de Paris devem apresentar planos de diminuição. Mas os planos de emissões ainda são eletivos e não impostos de fora, ou seja, cada membro adota uma meta propicia, conforme [35].

Em 2016, se apontou no planeta um ponto recorde de emissões de $\mathrm{CO}_{2}$, algo que peritos no tema, classificaram de "uma nova era" de aquecimento global e "prova irrefutável" da tarefa humanitária com relação as transformações ambientais. Os Estados Unidos colaboram com cerca de $15 \%$ das emissões globais de particulados de carbono, todavia simultaneamente é um considerável meio de patrocínio e inovação para países em desenvolvimento em seus empenhos para extinguir o aquecimento global, conforme [9]. A mitigação do crescimento da temperatura global é tema reafirmada pelas convenções de desenvolvimento sustentável ao longo do tempo, tornando o acordo de Paris continuidade sobre o tema de controle de emissões de gases e partículas poluidoras da atmosfera, por um modelo desenvolvimento sustentável, ou seja, o mundo precisa controlar o transporte desta massa poluidoras da atmosfera.

O acordo de Paris criou mecanismo sob o tema, no acordo os objetivos de mitigação do avanço da temperatura respeitando a limitação financeira de cada membro. Além disso, o acordo retrata a importância do acompanhamento das emissões antrópicas por fontes de gases poluidores e criação de medida domésticas para tratar sobre o tema, é de fundamental importância a sistematização dos dados de emissões de cada fonte antrópica. Pois, através destes dados possibilitam estudos de controle de emissões por fontes antrópicas, os modelos matemáticos são mecanismo, ou seja, para ferramentas de gestão ambiental [22-23], assim no próximo tópico será retratado uma breve revisão teórica sobre a importância dos modelos matemáticos com ferramentas de gestão ambiental.

\section{MODELOS MATEMÁTICOS MECANISMO DE GESTÃO AMIBIENTAL}

A investigação da dispersão de poluentes na atmosfera é uma atividade de fundamental importância para proteção da qualidade do ar. Nas últimas décadas, a dispersão de poluentes na atmosfera tornou-se assunto de grande relevância para a comunidade científica, devido ao aumento das emissões de poluentes ocasionadas pelo crescimento das áreas urbanas e industriais, para conforme[1,17,24-25]. Outro fator decisivo que tem levado ao estudo sistemático da dispersão de poluentes na atmosfera é a localização dos grandes centros urbanos e industriais. Em geral, estes centros estão localizados em regiões bastante heterogêneas em termos de relevo, tipos de solo e ocupação. Estas especificidades tornam mais difíceis à caracterização da dispersão de contaminantes. Como consequência da própria configuração do terreno podem surgir circulações locais que tornam ainda mais difícil a previsão da dispersão de contaminantes. Dessa maneira, uma melhor compreensão destes processos pode levar a um melhor controle e gerenciamento da qualidade do ar.

O cálculo da dispersão de poluentes leva em consideração diversos fatores; entre os mais importantes estão: velocidade média do vento, direção do vento, insolação, grau de estabilidade da atmosfera, temperatura da atmosfera, relevo, características das fontes poluidoras e dos gases sendo emitidos.

A dispersão, portanto, é um processo extremamente complexo. Isto é devido ao fato que, os escoamentos na baixa atmosfera, na chamada Camada Limite Planetária (CLP), são governados pela turbulência, cuja física ainda permanece longe de ser 
completamente compreendida. Portanto, os modelos para o cálculo da dispersão de poluentes devem ser capazes de simular em detalhes os efeitos da turbulência (flutuações do vento médio), para obter previsões confiáveis da concentração de contaminantes. Estes modelos variam em grau de complexidade, e podem ser classificados em duas principais classes: os Eulerianos e Lagrangeanos.

Os modelos Eulerianos utilizam soluções da equação de difusão-advecção, em um sistema de referência fixo em relação à terra. Os modelos Lagrangeanos simulam trajetórias de partículas de poluentes em um sistema de referência que acompanha o movimento das mesmas. Existem ainda outros modelos que podem ser considerados como subclasses destes primeiros [21]. Entre eles estão os modelos de pluma Gaussianos, que são os mais comuns, porém extremamente limitados. Eles se aplicam somente a situações de vento constante e turbulência homogênea (flutuações uniformes do vento médio, iguais em todas as direções), condições que dificilmente são encontradas na CLP. Modelos Gaussianos/Lagrangeanos do tipo puff, que são aperfeiçoamentos destes últimos, possuem uma aplicabilidade bem maior, com a vantagem de consumir, em média, um tempo de CPU 30 vezes menor do que os modelos de partículas Lagrangeanos. Nestes modelos, a pluma é representada por uma série de pacotes discretos (nuvens) de material poluente.

As informações meteorológicas variando no tempo, necessárias para os modelos de dispersão, incluindo os efeitos da turbulência podem vir de observações ou de saídas de modelos meteorológicos. Os modelos meteorológicos podem ser classificados ainda como diagnósticos ou prognósticos. Os modelos diagnósticos utilizam observações meteorológicas ou saídas de outros modelos para produzir campos de velocidade, temperatura e outras variáveis, necessárias aos modelos de dispersão. Isto é feito utilizando as equações de conservação, sem os termos de variação temporal, onde os campos produzidos obedecem, por exemplo, no caso dos campos de velocidade, à lei de conservação de massa.

Em resumo, estes modelos calculam a melhor solução para os campos das variáveis de interesse, de um determinado local, para um dado tempo, a partir das informações meteorológicas fornecidas. Em geral são utilizados procedimentos do tipo análise objetiva. Nestes procedimentos as informações meteorológicas disponíveis (observações) são extrapoladas para os volumes (pontos) da malha (grade) utilizando, basicamente, dois tipos de esquemas. No primeiro são utilizadas funções peso, e as variáveis dependentes, nos pontos de malha (grade), são funções da distância entre os pontos e as observações. No segundo, utilizam-se rotinas de análise variacional, onde uma ou mais relações de conservação são aplicadas para minimizar a variância da diferença entre os campos observados e analisados,[29].

No entanto, os resultados fornecidos por estes modelos vão depender da densidade das observações ou da resolução da malha para poder representar com precisão os detalhes do escoamento de interesse. Como os dados de observação na maioria das vezes são esparsos (no tempo e no espaço), e as malhas grosseiras, estes modelos nem sempre oferecem resultados confiáveis. Os modelos meteorológicos prognósticos, por sua vez, resolvem as equações de conservação da termofluidodinâmica (adaptadas para o sistema atmosférico), avançando-as no tempo e fornecendo os campos de velocidade, temperatura e outras variáveis. Nas últimas décadas, os grandes avanços nas técnicas de modelagem numérica da atmosfera tornaram possível prever, com alto grau de precisão, circulações atmosféricas para um tempo futuro de aproximadamente (24 h). Isto é obtido através da integração das equações governantes, utilizando métodos 
numéricos sofisticados, o que hoje é conhecido como previsão numérica do tempo. Com os avanços nas técnicas de solução e melhorias na representação dos processos físicos, é possível hoje em dia representar com um alto grau de precisão os fenômenos atmosféricos das mais diversas escalas de tempo e espaço. Os chamados modelos regionais de previsão do tempo são hoje em dia as ferramentas mais avançadas na representação destes fenômenos. Os modelos Regional Atmospheric Modeling System (RAMS), [29], NCAR/Penn State Mesoscale Model (MM5), [13], estão entre os mais conhecidos e utilizados, atualmente.

Desta forma, pode-se obter uma melhor representação da dispersão de poluentes. Uma grande variedade de estudos deste tipo, para diferentes condições de terreno e condições meteorológicas, vem sendo desenvolvidas e validadas através de observações. O resultado disto são os chamados sistemas de modelos (dispersion modeling system ou air quality system), ou seja, modelos de dispersão acoplados a modelos meteorológicos que podem ser utilizados para um monitoramento, ou até previsão, da qualidade do ar, com um grau maior de precisão. Os modelos California Photochemical Grid Model (CALGRID), [35], California Puff Model (CALPUFF), [32] são exemplos de modelos de dispersão que tem sido rotineiramente acoplado a modelos meteorológicos para o estudo e previsão da dispersão de poluentes na atmosfera.

\section{APLICAÇÃO DA GESTÃO DAS EMISSÕES NO PORTO DE ARATU}

\subsection{Breve apresentação do porto de Aratu}

"O Porto de Aratu, localizado na cidade de Candeias, Bahia, porto do Brasil situado na baía de Todos os Santos, localização GPS Latitude: $12^{\circ} 46$ '39" S, Longitude: 38²9'44" W.O". Unidade portuária do Complexo da Bahia com maior movimentação de cargas. A Codeba apostilou em 2017, uma nova marca anual na movimentação de carga, por toneladas, de 7,1 milhões. [6].

O produto movimentado divide-se em granéis sólidos, líquidos e gás. A infraestrutura de quatro terminais; terminal de produtos gasosos (TPG), com berço de 180 $\mathrm{m}$; terminal de granéis líquidos (TGL) com dois berços que perfazem $340 \mathrm{~m}$ e dois terminais de granéis sólidos (TGS), com três berços, numa extensão de $366 \mathrm{~m}$.

\subsection{Política e Agenda Ambiental}

\subsubsection{Política Ambiental}

A política ambiental está implantada nos procedimentos da Companhia das Docas do Estado da Bahia, se referindo como uma das atribuições corporativas. As suas práticas são conduzidas com base num Sistema Integrado de Meio Ambiente, Segurança e Saúde no Trabalho, instituída com o propósito de moldar e monitorar procedimentos e operações da organização tendo em vista a preservação ambiental, bem como a prevenção de episódios que resultem em prejuízos ao meio ambiente e a segurança dos seus funcionários e das comunidades localizadas em torno das suas unidades operacionais[...].

\subsubsection{Agenda Ambiental Institucional 2017 - 2020}

A organizacional firmou em 2012 sua Agenda Ambiental que está vigente, e atualizada ao longo do tempo pela Diretoria Executiva da CODEBA. Acordada com o compromisso institucional em conformidade com o Plano Nacional de Logística Portuária (PNLP), o Sistema de Planejamento Estratégico da Secretaria Nacional de Portos (SNP) e o Conselho Nacional do Meio Ambiente (CONAMA),[6]. 


\subsection{Mecanismo de Gestão Ambiental da CODEBA.}

A observação constante das emissões de gases e particulados no Porto de Aratu e entorno, a Companhia instalou três estações de monitoramento do ar, recolocou em operação uma quarta estação e estudada a possibilidade de instalar uma quinta estação. Os mecanismos integram a Rede de Monitoramento da Qualidade do Ar e Meteorologia (RMAR) entorno do zoneamento portuário, compreendendo a llha de Maré e zona portuária marítima região da Baia de Todos os Santos. Essa rede possui monitoramento contínuo e online, coletando dados de emissões e dados meteorológicos da região, sistematizando informações sobre os poluentes exigidos pelo CONAMA003/90.

Os relatórios de qualidade do ar são realizados conforme padrões ambientais da resolução CONAMA 003/90 ou valores de referência, quando não legislado no Brasil. Para, [22-23], a meteorologia tem um papel fundamental em relação à qualidade do ar. $\mathrm{Na}$ verdade são os eventos meteorológicos que guiam a dispersão de poluentes e a sua deposição no solo, uma vez que estão imersos na atmosfera.

Os modelos matemáticos de dispersão de poluentes na atmosfera são instrumentos técnicos indispensáveis para o controle da poluição atmosférica em escala local e regional. Os pré-processadores meteorológicos têm grande importância, e isto se deve ao fato da medida meteorológica no solo e em outras alturas, poderem ser calculadas sobre todo o domínio de simulação as variáveis meteorológicas necessárias para o cálculo do campo de concentração de poluentes. A maior parte dos modelos operativos para a estimativa da dispersão de gases e partículas na CLP utilizam esquemas simples da turbulência atmosférica expressa em classes de estabilidade como as propostas por [12]. Cada classe cobre um grande intervalo de condições de estabilidade atmosférica que resultam dependentes do lugar em que são avaliadas.

Nestes últimos anos abriu-se a possibilidade do desenvolvimento de modelos que descrevem a difusão dos poluentes que utilizam como entrada dados meteorológicos no solo (com possibilidade de serem adquiridos com uma rede meteorológica automática) que podem avaliar diretamente a turbulência atmosférica, pelo valor do comprimento de Monin-Obukhov, aplicado em 1954 e da velocidade de atrito, abrindo, assim, a perspectiva para modelos caracterizados por um maior conteúdo científico. Recentemente, o melhoramento da descrição e previsão dos fenômenos de poluição é devido ao aprimoramento dos modelos meteorológicos de mesoescala, que hoje podem trabalhar com resolução espacial melhor (grade mais refinada) e descrevem cenários sempre mais complexos.

\section{CONCLUSÃO}

Neste artigo foi abordado a utilização dos modelos matemáticos com mecanismos para gestão ambiental. O problema apresentado nessa pesquisa consistiu em vincular os pactos de gestão ambiental aos modelos matemáticos, pois na literatura os assuntos são abordados de forma separada.

Se referindo aos objetivos trabalhados no âmbito deste artigo, fundamentou a evolução dos pactos internacional de gestão ambiental, apresentou fundamentos da importância dos modelos matemáticos para gestão ambiental e por fim, apresentou a aplicação da gestão ambiental no Porto de Aratu no município de Candeias Bahia.

\section{Agradecimentos}

Os autores agradecem ao SENAI CIMATEC e à Fundação de Amparo à Pesquisa do Estado da Bahia (FAPESB) pelo apoio financeiro. 


\section{REFERÊNCIAS}

${ }_{1}$ ACIOLI, P.S.; XAVIER, F.A.; MOREIRA, D.M. Mathematical Model Using Fractional Derivatives Applied to the Dispersion of Pollutants in the Planetary Boundary Layer. Boundary-layer meteorology, v. 170, n. 2, p. 285-304, 2019.

2ALMEIDA, J. R. Avaliação de impacto ambiental em uma mata ciliar na cidade de Manaus. Revista Internacional de Ciências - RIC, Rio de Janeiro, v. 1, n. 1, p. 1 - 16, jan/jun.2011.

3BARBOSA, G.S. O desafio do desenvolvimento sustentável. Revista Visões, v. 4, n. 1, p. 1-11, 2008.

4BOULIC, R.; RENAULT, O. "3D Hierarchies for Animation”, In: New Trends in Animation and Visualization, Edited by Nadia Magnenat-Thalmann and Daniel Thalmann, John Wiley \& Sons Itd., England, 1991.

5BRÜSEKE, J.F. O problema do desenvolvimento sustentável. In: Desenvolvimento e Natureza: estudos para uma sociedade sustentável. CAVALCANTI, Clóvis (org.) - 2a ed. - São Paulo: Cortez; Recife, PE: Fundação Joaquim Nabuco, 1998.

${ }_{6}$ CODEBA - Companhia das Docas do Estado da Bahia. Disponível em: http://www.codeba.com.br/eficiente/sites/portalcodeba/pt-br/home.php. Acesso em: novembro/ 2018.

7Charge. Disponível em: http://www.charge-o-matic.blogger.com.br/charge-do-Benett.gif. Acesso em: Acesso em: junho/ 2019

8DE PASSOS, P.N.C. A conferência de Estocolmo como ponto de partida para a proteção internacional do meio ambiente. Revista Direitos Fundamentais \& Democracia, v. 6, n. 6, 2009.

9 DENNY, D.M.T. AGENDA 2030 GOVERNANÇA AMBIENTAL ESTUDO DE CASO BONSUCRO, 2017.

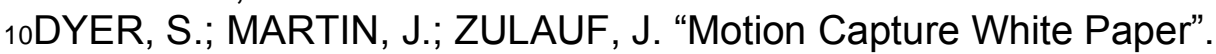

Disponível: $\quad$ http://reality.sgi.com/employees/jam_sb/mocap/MoCapWP_v2.0.html, Dezembro/1995. Acesso em: junho/ 2019

${ }_{11} \mathrm{GIEHL}, \mathrm{G}$. O direito ambiental e a importância da água. In: Âmbito Jurídico, Rio Grande, IX, n.36, jan2007. Disponível em: http://www.ambitojuridico.com.br/site/index.phpn_link=revista_artigos_leitura\&artigo_id= 1635. Acesso em: 19 jun 2019.

${ }_{12}$ GIFFORD JR, F. A. Use of routine meteorological observations for estimating atmospheric dispersion. Nuclear Safety, v. 2, p. 47-51, 1961.

${ }_{13}$ GRELL, G.A. et al. A description of the fifth-generation Penn State/NCAR mesoscale model (MM5). 1994.

14GUIMARÃES, R.P.; DA FONTOURA, Y.S dos R. Rio+ 20 ou Rio-20?: crônica de um fracasso anunciado. Ambiente \& Sociedade, v. 15, n. 3, p. 19-39, 2012.

${ }_{15} \mathrm{HOLTON}, \mathrm{M}$; ALEXANDER, S. "Soft Cellular Modeling: A Technique for the Simulation of Non-rigid Materials", Computer Graphics: Developments in Virtual ${ }_{16}$ Environments, R. A. Earnshaw and J. A. Vince, England, Academic Press Ltd., p. 449460, 1995.

17KITAGAWA, Y.K. L. et al. Evaluation of the chemical transport of air pollutants in the metropolitan region of Salvador, Brazil. WIT Transactions on Ecology and the Environment, v. 230, p. 519-530, 2018.

${ }_{18} \mathrm{KNUTH}, \mathrm{D}$. E. The TEXBOOK, Addison Wesley, 15th edition, 1984.

${ }_{19} \mathrm{MAIA}$, J. et el. Modelo matemático para avaliação de impacto ambiental (AIA) Mathematical model for environmental impact assessment (AIA). Revista Brasileira de 
Gestão Ambiental. Artigo Científico. Disponível em: http://www.gvaa.com.br/revista/index.php/RBGA/index . Acesso abril/ 2019.

20MALHEIROS, T.F.; PHLIPPI JR, A.; COUTINHO, S. M. V. Agenda 21 nacional e indicadores de desenvolvimento sustentável: contexto brasileiro. Saúde e Sociedade, v. 17, p. 7-20, 2008.

21LONGHETTO, A. Atmospheric planetary boundary layer physics: proceedings of the 4th Course of the International School of Atmosperic Physics," Ettore Majorana" Centre for Scientiic Culture, held in Erice (Italy), 13-27 February 1978. Elsevier Science \& Technology, 1980.

22MOREIRA, D.M; TIRABASSI, T. Modelo matemático de dispersão de poluentes na atmosfera: um instrumento técnico para a gestão ambiental. Ambiente \& sociedade, v. 7, n. 2, 2004.

23MOREIRA, D.M; TIRABASSI, T.; DE MORAES, M.R. Meteorologia e poluição atmosférica. Ambiente \& Sociedade, v. 11, n. 1, p. 1-13, 2008.

24MOREIRA, D.M. e MORET, M., 2018 A new direction in the atmospheric pollutant dispersion inside of the Planetary Boundary Layer. J. Appl. Meteor. Climatology 57(1), 185-192.

25MOREIRA, D. M.; SANTOS, 2019. New approach to handle gas-particle transformation in air pollution modelling using fractional derivatives. Atmospheric Pollution Research, In press.

${ }_{26}$ PASQUILL, F.; SMITH, F. B. Atmospheric diffusion. New York: Wiley, 1983. p. 437 ${ }_{27}$ PENEREIRO, J. L.; FERREIRA, D. H. L. A modelagem matemática aplicada às questões ambientais: uma abordagem didática no estudo da precipitação pluviométrica e da vazão de rios. Revista Millenium, Coimbra, n. 42, p. 27 - 47, 2012. Disponível em: http://www.revistaea.org/pf.php?idartigo=1626 Acesso em: abril/ 2019.

${ }_{28} \mathrm{OOKOUCHI}, \mathrm{Y}$. et al. Avaliação dos efeitos da umidade do solo na geração e modificação de circulações em mesoescala. Revisão meteorológica mensal , v. 112, n. 11, p. 2281-2292, 1984.

${ }_{29}$ PIELKE, R.A. et al. A comprehensive meteorological modeling system-RAMS. Meteorology and atmospheric Physics, v. 49, n. 1-4, p. 69-91, 1992.

30REIS, F. A. G. V.; GIORDANO L. C.; CERRI, L. E. S.; MEDEIROS, G. A. Contextualização dos cursos superiores de Meio Ambiente no Brasil: Engenharia Ambiental, Engenharia Sanitária, Ecologia, Tecnólogos e Sequências. Espírito Santo do Pinhal, jan/dez 2005.

${ }_{31}$ RODRIGUES, J. P. Modelagem matemática da dispersão aplicada à definição de locais para o monitoramento da qualidade do ar. 2013. 74 p. Monografia (Trabalho de Conclusão de Curso - Curso Superior de Tecnologia em Processos Ambientais). Universidade Tecnológica Federal do Paraná. 2013.

${ }_{32}$ DA MOTTA, R. S. Analyzing the environmental performance of the Brazilian industrial sector. Ecological Economics, v. 57, n. 2, p. 269-281, 2006.

${ }_{33} \mathrm{SCIRE}$, Joseph S. et al. Um guia do usuário para o modelo de dispersão

CALPUFF. Earth Tech, Inc. Concord, MA , v. 10, 2000.

${ }_{34} \mathrm{SMITH}, \mathrm{A}$; ; JONES, B. On the complexity of computing. In Advances in Computer Science, pages 555-566. Publishing Press, 1999.

${ }_{35}$ SOUZA, M.C.O; CORAZZA, R.I. Do Protocolo Kyoto ao Acordo de Paris: uma análise das mudanças no regime climático global a partir do estudo da evolução de perfis de emissões de gases de efeito estufa. Desenvolvimento e Meio Ambiente, v. 42, 2017. ${ }_{36}$ XAVIER, P.H.F.; NASCIMENTO, E.G.S.; MOREIRA, D.M., 2018. A model using fractional derivatives with vertical eddy diffusivity depending on the source distance 
applied to the dispersion of atmospheric pollutants. Pure and Applied Geophysics 176, 1797-1806.

${ }_{37}$ YAMARTINO, R. J. et al. The CALGRID mesoscale photochemical grid model-I. Model formulation. Atmospheric Environment. Part A. General Topics, v. 26, n. 8, p. 14931512, 1992. 